\title{
Nuclear Drosha enhances cell invasion via an EGFR-ERK1/2-MMP7 signaling pathway induced by dysregulated miRNA-622/197 and their targets LAMC2 and CD82 in gastric cancer
}

\author{
Liyun $\mathrm{Xu}^{1,4}$, Yixuan Hou ${ }^{1,2,4}$, Gang Tu${ }^{3}$, Yanlin Chen ${ }^{1}$, Yan-e Du${ }^{1}$, Hailong Zhang ${ }^{1}$, Siyang Wen ${ }^{1}$, Xi Tang ${ }^{1}$, Jiali Yin ${ }^{1}$, Lei Lang ${ }^{1}$, \\ Kexin Sun ${ }^{1}$, Guanglun Yang ${ }^{3}$, Xiaoli Tang ${ }^{1}$ and Manran Liu ${ }^{\star, 1}$
}

Drosha is an RNA III-like enzyme that has an aberrant expression in some tumors. Our previous studies showed the aberrant Drosha in gastric tumors. However, the roles of nuclear Drosha, the main regulator of microRNA (miRNA) biogenesis, in gastric cancer (GC) progression remain poorly understood. In this study, we demonstrated that nuclear Drosha is significantly associated with cell invasion of GC and that Drosha silence impedes the tumor invasion. Knockdown of Drosha led to a set of dysregulated miRNAs in GC cells. Multiple targets of these miRNAs were the members in cell migration, invasion and metastasis-associated signaling (e.g. ECM-receptor interaction, focal adhesion, p53 signaling and MAPK signaling pathway) revealed by bioinformatics analysis. LAMC2 (a key element of ECM-receptor signaling) and CD82 (a suppressor of p53 signaling) are the targets of miR-622 and miR-197, respectively. High levels of LAMC2 and low levels of CD82 were significantly related to the worse outcome for GC patients. Furthermore, overexpression of LAMC2 and knockdown of CD82 markedly promoted GC cell invasion and activated EGFR/ERK1/2-MMP7 signaling via upregulation of the expression of phosphorylated (p)-EGFR, p-ERK1/2 and MMP7. Our findings suggest that nuclear Drosha potentially has a role in the development of GC.

Cell Death and Disease (2017) 8, e2642; doi:10.1038/cddis.2017.5; published online 2 March 2017

Gastric cancer (GC) is the most common gastrointestinal cancer with high morbidity and mortality in China. There are $\sim 740000$ deaths each year, accounting for $10 \%$ of total cancer death. ${ }^{1}$ Tumor invasion and metastasis are the major cause for the high mortality rate of GC. Recently, multiple molecular alterations, such as the activation and overexpression of oncogenic $R A S{ }^{2}$ inactivation of tumor suppressor genes $p 53^{3}$ and even some of the noncoding $\mathrm{RNA}^{4}$ have been shown to be involved in gastric carcinoma metastasis. However, the mechanism of gastric tumor metastasis is not fully understood.

Drosha is an enzyme of endonuclease RNase III, which is critical for the canonical microRNA (miRNAs) biogenesis. Aberrant expressions of Drosha are closely related to carcinogenesis and cancer progression. However, there are controversial reports about Drosha expressions in different human malignancies. For example, the downregulated Drosha proteins were detected in gallbladder cancer and the decreased Drosha was used as indicators of poor prognosis in gallbladder cancer patients. ${ }^{5}$ In contrast, the increased Drosha expressions were observed in non-small-cell lung cancer, ${ }^{6}$ which was closely related to the pathological stage, tumor metastasis and worse prognosis. It is inseparable that the role of aberrant Drosha in the tumor progression may be dependent on miRNAs, which have referred to tumor initiation and development as oncogenes or tumor suppressor genes through negative regulation of hundreds of target genes at the post-transcriptional level. ${ }^{7}$ Dysregulated miRNAs are detected in different kinds of cancers, including colorectal carcinoma, ${ }^{8}$ breast cancer ${ }^{9}$ and $\mathrm{GC},{ }^{4}$ and involved in tumor pathology, diagnosis, treatment, prognosis and other processes. For example, miR-21 inhibits lung squamous carcinoma cell proliferation and metastasis by targeting PTEN and RECK. ${ }^{10}$

Our previous studies have shown that the aberrant Drosha expression may be associated with tumor malignancy in GC. ${ }^{11}$ However, the roles of Drosha in human GC remains to be elucidated. In this study, we further analyzed Drosha expression in gastric tumor tissues and their adjacent normal tissues by immunohistochemistry (IHC) and qRT-PCR. The silence of Drosha expression using interfering RNA in GC led to impeded tumor cell invasion and change of miRNA profiles. Knockdown of Drosha significantly reduced cell invasion via an EGFR-ERK1/2-MMP7 signaling pathway, which is partly due to miR-622 and miR-197 targeting LAMC2 and CD82, respectively. Thus, our study provides a mechanistic insight into the function of Drosha in gastric metastasis via an altered miRNA profile.

\footnotetext{
${ }^{1}$ Key Laboratory of Laboratory Medical Diagnostics, Chinese Ministry of Education, Chongqing Medical University, Chongqing 400016, China; ${ }^{2}$ Experimental Teaching Center of Basic Medicine Science, Chongqing Medical University, Chongqing 400016, China and ${ }^{3}$ Department of Endocrine and Breast Surgery, The First Affiliated Hospital of Chongqing Medical University, Chongqing 400016, China

*Corresponding author: M Liu, Key Laboratory of Laboratory Medical Diagnostics, Chinese Ministry of Education, Chongqing Medical University, No. 1, Yi-Xue-Yuan Road, Yu-zhong District, Chongqing 400016, China. Tel: +86 23 68485938; Fax: +86 23 68485239; E-mail: mliu-hncq@ @otmail.com

${ }^{4}$ These authors contributed equally to this work.

Received 09.8.16; revised 10.12.16; accepted 13.12.16; Edited by E Candi
} 


\section{Results}

Drosha expression in GC tissues and cell lines. Our previous studies have shown that aberrant nuclear Drosha was upregulated in GC. ${ }^{11}$ To understand whether high levels of nuclear Drosha are a bad predictor for patients with GC, we further detected Drosha expression in gastric tumor tissues by IHC staining. Consistent with our previous findings, the nuclear Drosha was significantly higher in gastric adenocarcinoma than that in the tumor in situ (preinvasive tumor, PT) and normal gastric tissues (data are not shown). Compared with PTs, the gradually enhanced nuclear Drosha proteins were detected in lymph node metastasis tissues (N0-N3) and distant metastasis tissues (M) (Figures 1a and b). The similar mRNA expression patterns of Drosha were disclosed in these tissues (Figure 1c). To further verify this association of the Drosha expression pattern and the malignancy of GC, the expressions and distribution of Drosha were assessed in four of the poorly differentiated GC cells (MKN-28, NUGC-3, BGC-803 and HGC-27) and the well-differentiated GC cell (NCL-87) by western blot and immunofluorescence (IF) staining; as expected, the enhanced nuclear Drosha was observed in malignant GC cells (Figures 1d and e). These data indicate that the high levels of nuclear Drosha may associate with GC metastasis.

Drosha silence reduces cell migration potential of GC cells. To further understand the roles of Drosha in GC metastasis, siRNA interference of Drosha expression was used. Drosha was verified to be efficiently knocked down by shRNA against Drosha in MGC-803 GC cells (Figure 2a). Thus, the lentivirus-mediated shRNA 2\# and shRNA 3 \# were stably infected into GC MGC-803, NUGC-3 and HGC-27 cells. Efficiency of knocking down Drosha (Figures $2 \mathrm{~b}$ and c) led to a clear slowdown of motility ability (Figure $2 \mathrm{~d}$ ) and invasive potentials of MGC-803 and NUGC-3 cells (Figure 2e). These data suggest that Drosha may have a role in promoting migration and invasion of GC cells.

miRNA profiles were dysregulated in Drosha-silenced GC cells. It has been known that Drosha has an important role in the canonical miRNA biogenesis in the nucleus. Thus, we guessed that some of the miRNAs and their target genes associated with cell migration and invasion may respond to dysregulated Drosha in the GC. Indeed, a set of miRNAs (47 upregulated and 14 downregulated) were identified in Drosha-knockdown MGC-803 cells by miRNA array analysis (Figure 3a). To validate the miRNA assay data, eight of the randomly chosen dysregulated miRNAs were tested by qRT-PCR in MGC-803 and NUGC-3 cells (Figure 3b). Using TargetScan v.6.2, miRanda and DIANA-microT, a total of 4088 subsequent function mRNA targets was predicted. The significantly altered signaling pathways $(P<0.05)$ were compiled using DAVID v.6.7, in which ECM-receptor signaling pathway, p53 signaling pathway, focal adhesion signaling, MAPK signaling pathway, TGF- $\beta$ signaling pathway and mTOR signaling pathway were reported to be related to cell migration, invasion and metastasis (Figure $3 c$ ).
miR-622 and miR-197, respectively, direct target LAMC2 and CD82. Next, we wondered which key miRNAs and their associated targets or signaling pathways may have critical roles in GC metastasis. Twenty of the top changed miRNAs in our miRNA array, their functional targets and corresponding signaling pathways were carefully analyzed by bioinformatics or paper reviewing. The $L A M C 2$ and $C D 82$ were found to be closely related with ECM-receptor signaling pathway or p53 signaling pathway, which was suggested to contribute to tumor metastasis. Six of these miRNAs, including miR-622, miR-197, miR-199b-5p, miR-146a, miR-129 and miR-130-5p, may be the regulators of $L A M C 2$ and $C D 82$. Of these, miR-622 and miR-197 have a high score to bind to the $3^{\prime}$-UTR of LAMC2 or CD82, respectively (Figure $4 \mathrm{a}$ ). These were further verified by luciferase assay. LAMC2 was suppressed by miR-622 and $C D 82$ was repressed by miR-197. In addition, mutation of the binding sites in the $3^{\prime}$-UTRs of LAMC2 or CD82 canceled the responsiveness of these genes to ectopic miR-622 or miR-197 in MGC-803 cells (Figure 4b). Similarly, the endogenous mRNA levels of LAMC2 in MGC-803 cells were significantly decreased under overexpression of miR-622. The levels of LAMC2 had no significant change when miR-622 was knocked down by its specific shRNA (Figure 4c, left panel). On the other hand, there was no much change of endogenous CD82 after it was transfected with miR-197 in MGC-803 cells. However, the mRNA of CD82 was markedly enhanced after knockdown of miR-197 using shRNA in MGC-803 cells (Figure 4c, right panel). Knocking down Drosha in MGC-803 and NUGC-3 cells, the decreased LAMC2 or increased CD82 was detected in mRNA and protein levels (Figures $4 d$ and e). These data indicate that $L A M C 2$ is a major target of miR-622, and CD82, a major target of miR-197.

\begin{abstract}
Aberrant LAMC2 and CD82 expressions are associated with bad outcome for GC patients. To characterize the association of LAMC2 and CD82 with the prognosis of GC, the Kaplan-Meier survival curves were applied using 876 GC patients in the KM plots database (http: //www.kmplot.com), and GC patients with higher levels of LAMC2 and/or lower levels of CD82 had longer survival (Figure 5a). Consistently, higher levels of LAMC2 and lower levels of CD82 had a significant effect on cell invasion and metastasis of GC revealed by $\mathrm{IHC}$ staining (Figures $5 \mathrm{~b}$ and $\mathrm{c}, P<0.01$ ) and qRT-PCR analysis (Figures $5 d$ and e, $P<0.01$ ). Taken together, these data demonstrate that higher levels of LAMC2 and lower levels of CD82 were significantly associated with $\mathrm{GC}$ invasion and metastasis.
\end{abstract}

\section{LAMC2 promotes and CD82 suppresses GC invasion via an EGFR/ERK1/2-MMP7 signaling pathway. LAMC2} could colocalize with EGFR in the ATC cells ${ }^{12}$ and CD82 suppresses the phosphorylation of EGFR in EGF- and HGFdependent manner in Hepa1-6 cells, ${ }^{13}$ indicating that the aberrant LAMC2 and CD82 may be involved in tumor cell invasion through the activity of EGFR and its downstream signaling in GC cells. Indeed, the activities of EGFR and its downstream ERK1/2 signaling were repressed, and expression of MMP7 was decreased in Drosha-silenced MGC-803 and NUGC-3 cells (Figure 6a). To further confirm these 
a

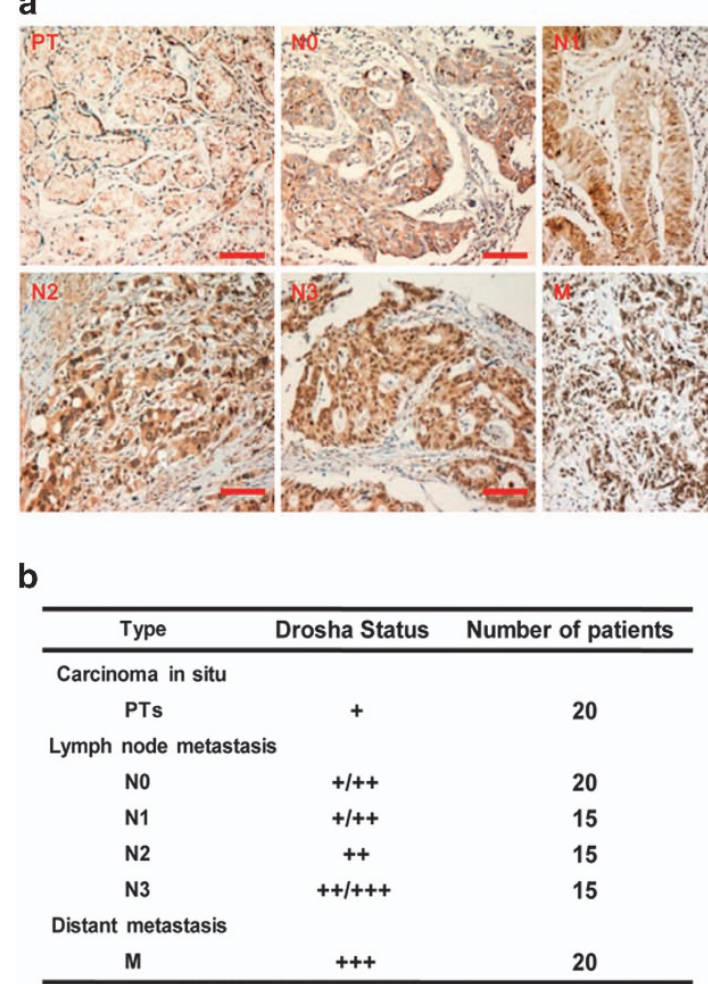

C

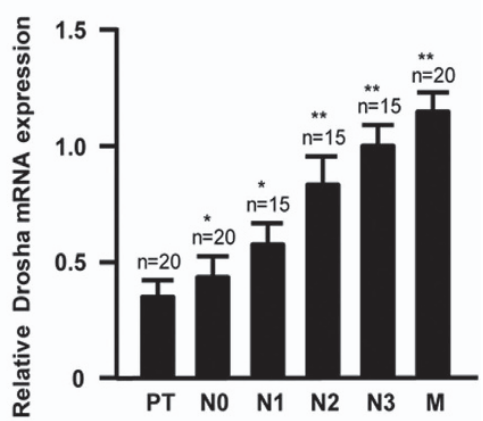

e

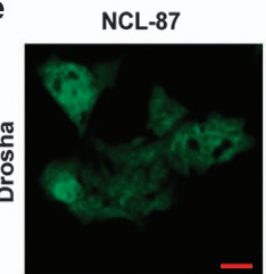

MKN-28

d
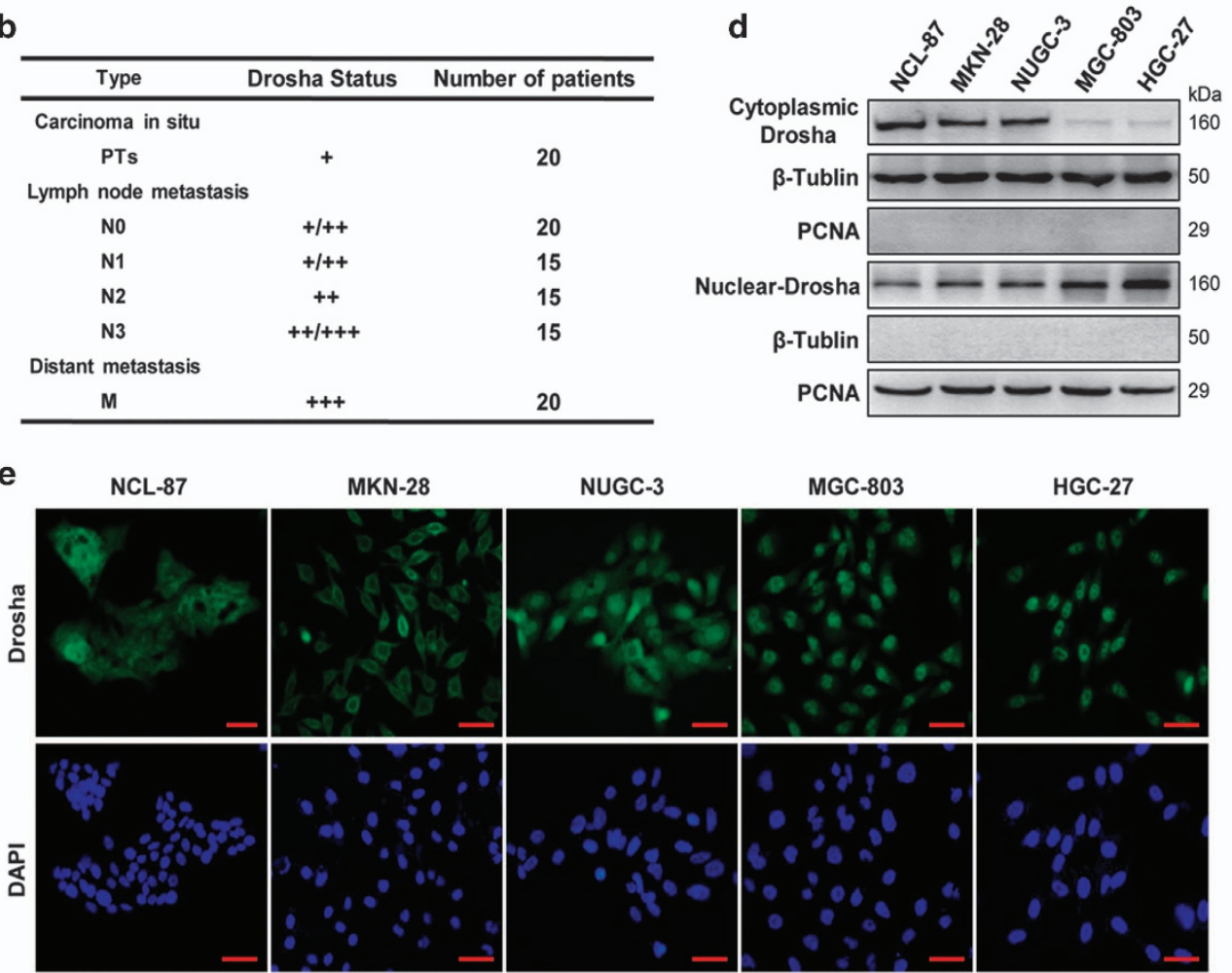

MGC-803

HGC-27
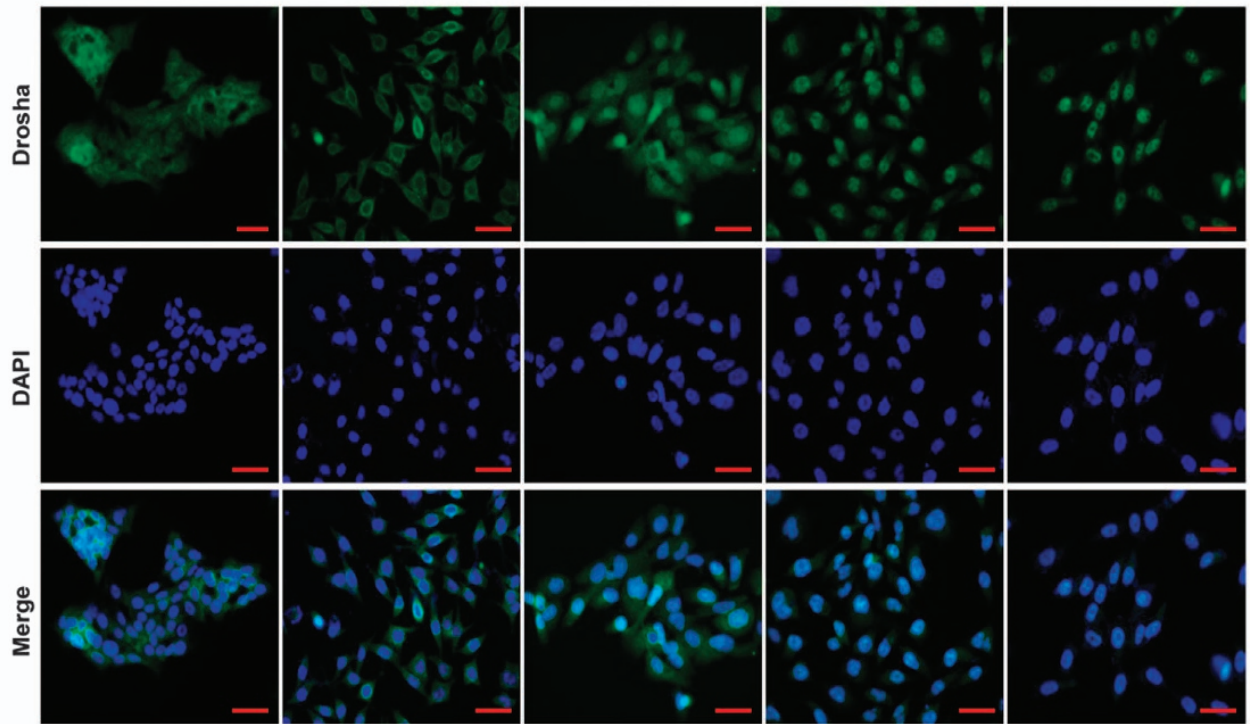

Figure 1 Drosha expression in GC tissues and cell lines. (a) Representative images of Drosha staining in the tumor in situ (PT), lymph node metastasis tissues (NO-N3) and distant metastasis tissues (M). Scale bars, $100 \mu \mathrm{m}$. (b) Summary of IHC staining for carcinoma in situ (PT), lymph node metastasis (N0-N3) and distant metastasis (M) GC samples. The nuclear staining intensities were categorized as low (+), medium (++) or high (+++) based on observations of $80 \%$ of the cell population. (c) Quantitation of Drosha mRNA was measured by qRT-PCR. The data represent means \pm S.D. from triplicate samples ( ${ }^{\star} P<0.05,{ }^{* \star} P<0.01$ versus PT). (d) Cytoplasmic and nuclear Drosha levels were determined by western blot in indicated GC cells. $\beta$-Tubulin: cytoplasmic protein marker; PCNA: nuclear marker. (e) Expression and localization of Drosha by IF staining in GC cells. Scale bars, $50 \mu \mathrm{m}$

findings, the specific shRNA against $L A M C 2$ or the miR-622 mimics was stably or transiently transfected into MGC-803 cells. The activities of EGFR and ERK $1 / 2$ were suppressed, and MMP7 expression was reduced after LAMC2 interfered
(Figure 6b). Correspondingly, tumor cell-invasive potentials were decreased in these cells (Figure $6 \mathrm{~d}$ ). The similar results were observed after rescuing CD82 expression by stably transfecting pcDNA-CD82 or sh/miR-197 into MGC-803 cells 
a

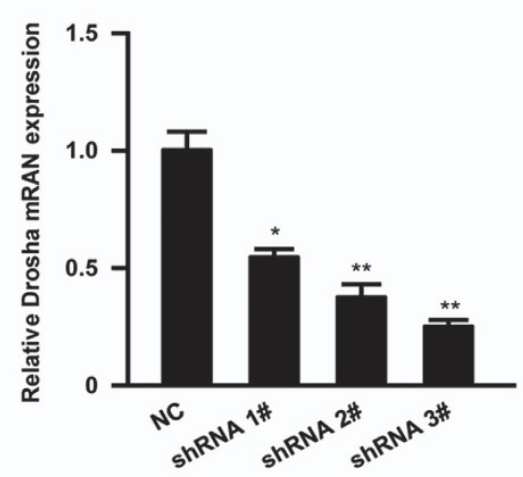

C

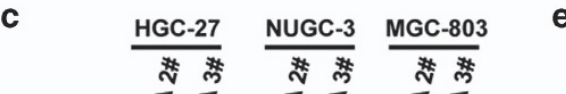

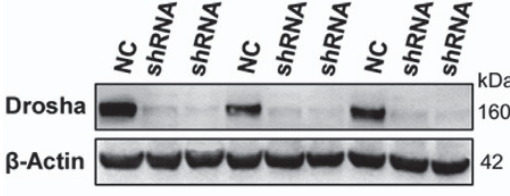

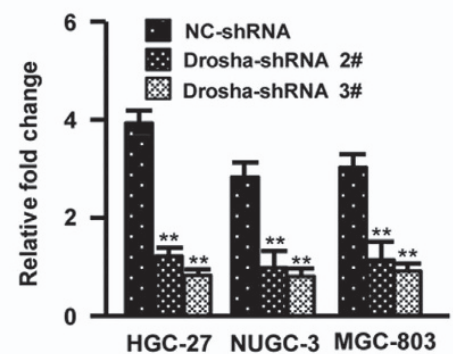

d

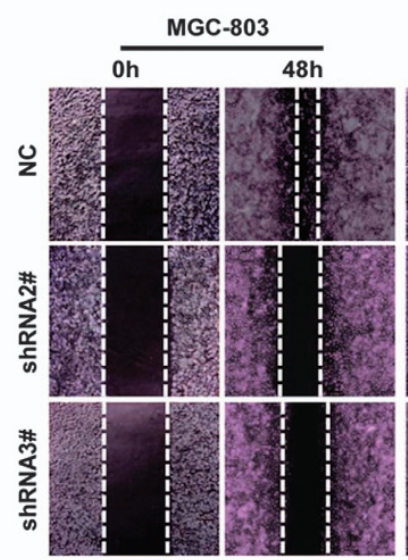

\section{b}

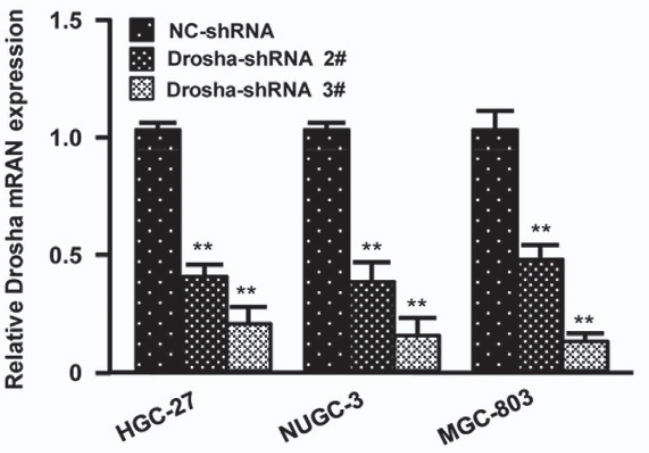

e
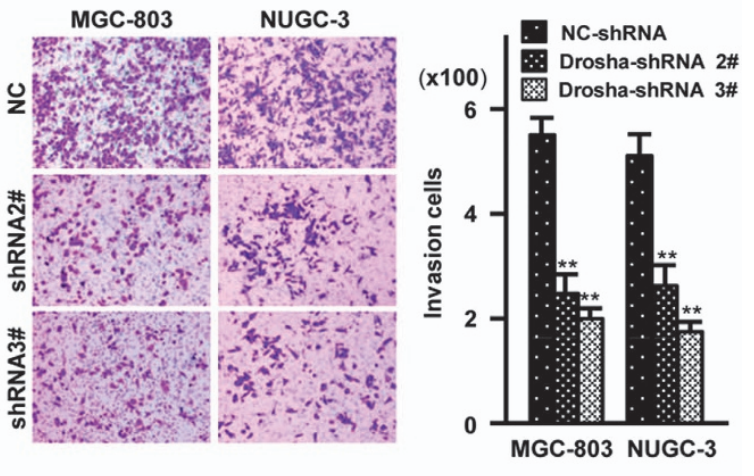

0 MGC-803 NUGC-3

HGC-27 NUGC-3 MGC-803
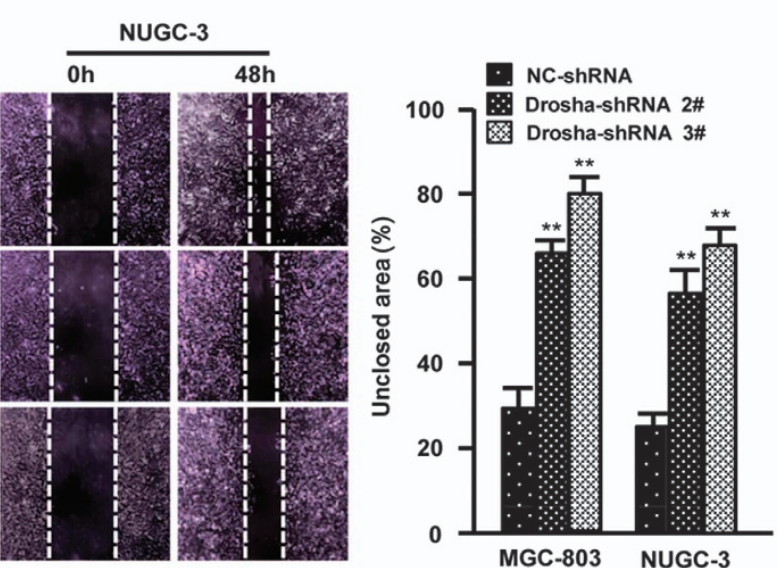

Figure 2 Drosha silence inhibits GC cell invasion. (a) The efficiency of short hairpin RNAs (shRNAs) against Drosha in 293T cells was determined by qRT-PCR ( ${ }^{*} P<0.05$, ${ }^{\star *} P<0.01$ versus control shRNA). (b and $\mathbf{c}$ ) Interference of Drosha in HGC-27, NUGC-3 and MGC-803 GC cells was detected by qRT-PCR (b) and western blotting (c) $\left({ }^{* *} P<0.01\right.$ versus control shRNA). $\beta$-Actin works as a loading control. ( $\mathbf{d}$ and $\left.\mathbf{e}\right)$ Cell mobility and invasion were tested by wound healing assay (d) or Transwell assay (e) for indicated GC cells with specific shRNA (2\# or 3\#) against Drosha. The mobility ability was determined as the percentage of non-healed scratched area by using the Image $\mathrm{J}$ software. The data are presented as the mean \pm S.D. $\left(n=3 ;{ }^{*} P<0.01\right.$ versus control shRNA)

(Figures 6c and e). Furthermore, the restoration of LAMC expression in Drosha-silenced MGC-803 cells led to the enhanced phosphorylation of EGFR and ERK1/2, and high levels of MMP7 (Figure 6f). As a result, the potential of tumor cell invasion was increased (Figure 6h). In line with this, knockdown of CD82 expression by sh/CD82 or miR-197 in
Drosha-silenced MGC-803 cells resulted in the increased expression of p-EGFR, p-ERK1/2 and MMP7 (Figure 6g), which rendered the tumor cells with a marked invasive potential (Figure 6i). These data suggest that the EGFR/ ERK1/2-MMP7 signaling pathway is the core in LAMC2promoting and CD82-suppressing GC cell invasion. 
a
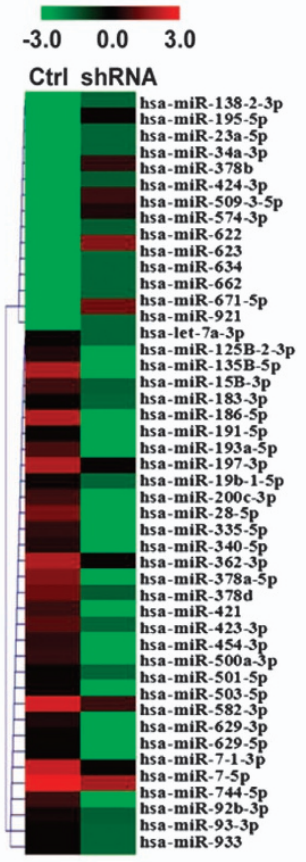

C

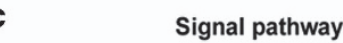

b

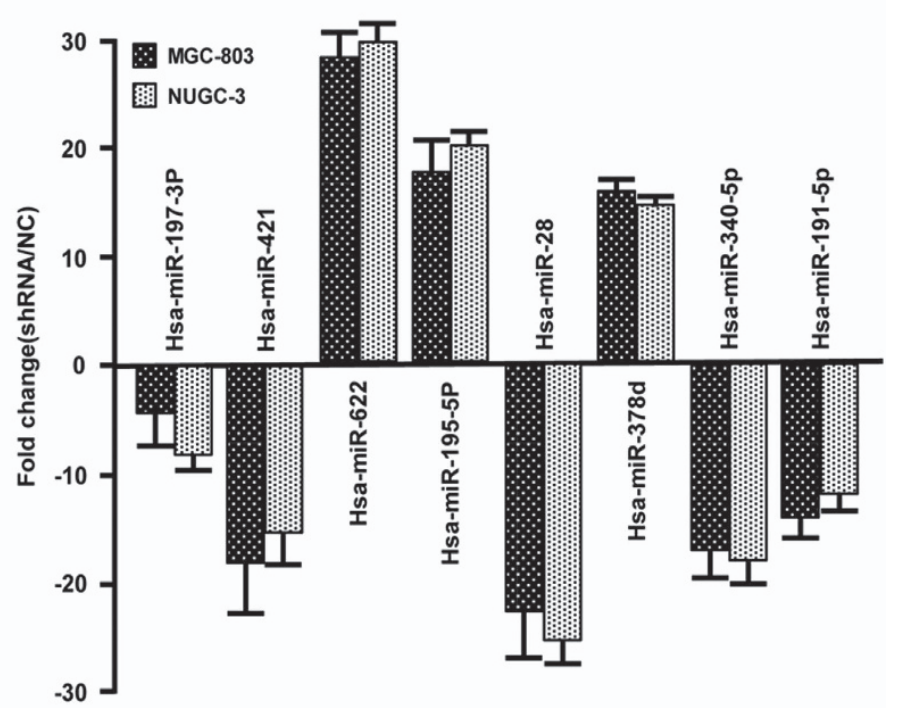

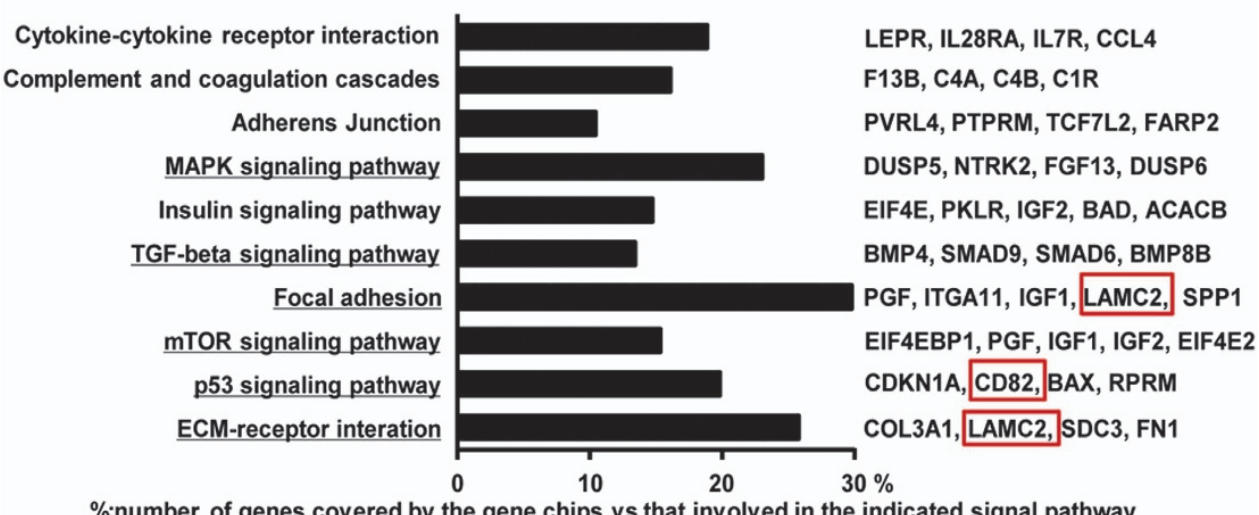

$\%:$ number of genes covered by the gene chips vs that involved in the indicated signal pathway

Figure 3 Global changes of miRNA profile in Drosha-silenced GC cells. (a) The heatmap of 47 altered miRNAs identified by miRNA array in Drosha-knockdown cells. (b) qRT-PCR to certify the altered miRNAs for the cumulative microarray data. (c) The major changed pathways $(P<0.05)$ of copredicted miRNA targets were enriched by DAVID v.6.7. The black column represents the number of copredicted miRNA target genes located on the pathway. The representative target genes and the interesting targets were shown on the right panel. Percentage $(\%)=$ the target genes/the total genes in a given pathway

\section{Discussion}

Drosha is a nuclear enzyme that cleaves the primary miRNAs to precursor miRNAs in the classic miRNA biosynthesis. ${ }^{14}$ Our previous studies have shown that the aberrant nuclear Drosha may be associated with tumor malignancy and differentiation in GC. ${ }^{11}$ However, the biological function of Drosha in GC was still unclear. Here we disclosed that the nuclear Drosha promotes tumor cell invasion in malignant GC cells through aberrant miRNA biosynthesis, and thus regulates their targets and corresponding signaling pathways.

Aberrant Drosha expressions have even been reported to closely associate with some solid tumor progression. Interestingly, the increased Drosha was usually related to pathologic features of the tumor, metastasis and prognosis in non-small-cell lung cancer. ${ }^{6}$ However, the enhanced nuclear Drosha was inversely correlated with tumor grade (breast cancer) ${ }^{15}$ or tumor invasion (e.g. human cutaneous melanoma), ${ }^{16}$ suggesting that the different function of Drosha may be based on different tumor types.

We found that nuclear Drosha has a key role in GC cell invasion. Higher levels of nuclear Drosha were detected in invasive and metastatic gastric carcinoma and cancer cells. Knockdown of Drosha expression decreased tumor cell invasion in the detected GC cells with higher nuclear Drosha. Using miRNA microarray and bioinformatics, most of the miRNA targets that were found to be the key members belonged to signaling pathways, which are associated with cell 
a

5sa-miR-622 3'...CGAGGUUGGAGUCGUCUGACA...5'
CD82 3'-UTR Position 452-458

5'....CCAUCCUgGCUAACAUgGUgaAA....3' || |||l| b
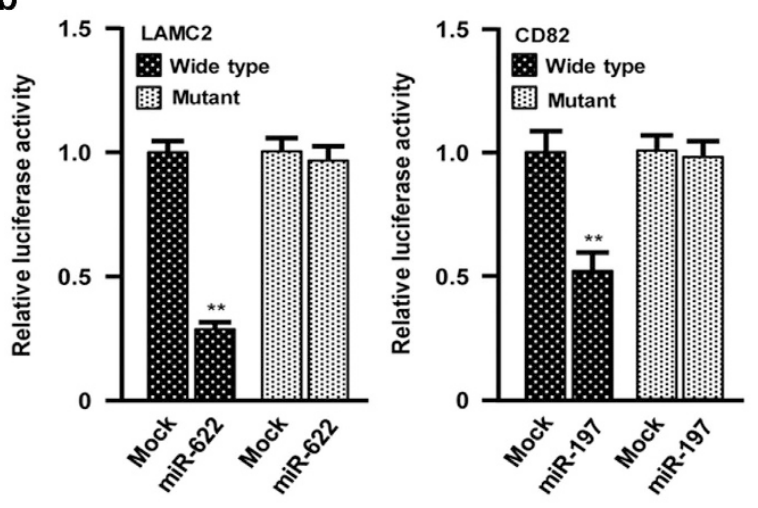

d

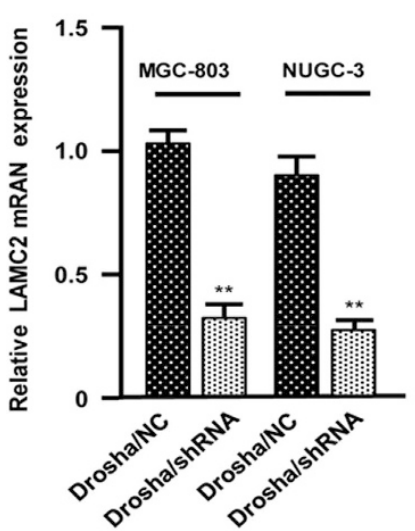

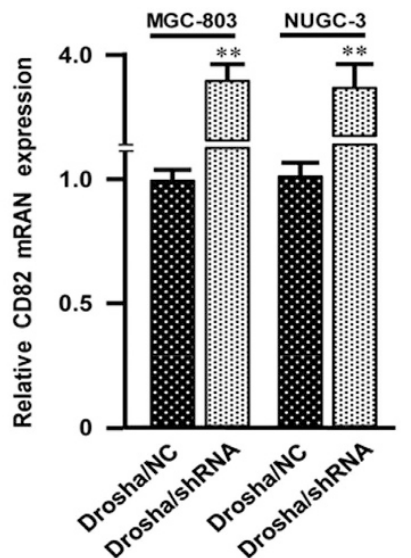

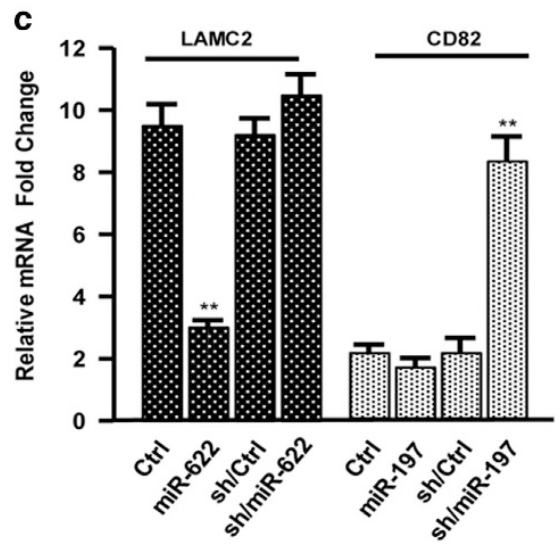

e

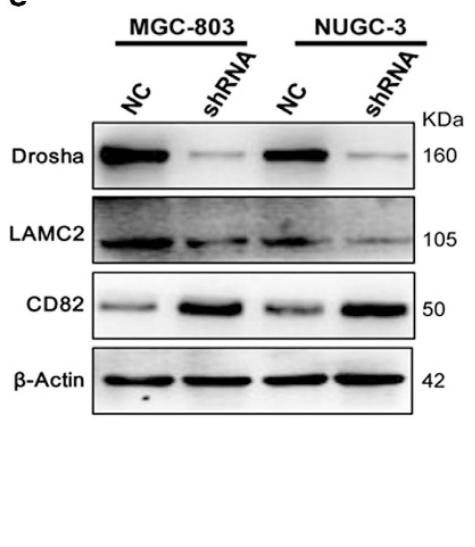

Figure 4 LAMC2 is a direct target of miR-622 and CD82 is a target of miR-197. (a) The predicted binding sites of miR-622 and miR-197 in LAMC2 and CD82 3'-UTRs. (b) Luciferase activities of LAMC2 and CD82 were tested in MGC-803 cells co-transfected with miR-622 or miR-197 mimics and their mimics control with wild-type (WT)/Mut 3'UTR of LAMC2 (left panel) or CD82 (right panel). The mean \pm S.D. represents three independent experiments ( ${ }^{\star *} P<0.01$ versus mock). (c) qRT-PCR to test the endogenous mRNAs of LAMC2 and CD82 in the indicated cells ( ${ }^{* *} P<0.01$ versus control). (d and $\left.\mathbf{e}\right)$ The indicated mRNAs (d) and proteins (e) were analyzed by qRT-PCR and western blotting assay in Drosha WT and silenced MGC-803 and NUGC-3 cells. $\beta$-Actin is the loading control $\left({ }^{* *} P<0.01\right.$ versus control shRNA cells)

invasion and cancer metastasis, such as ECM-receptor signaling pathway, p53 signaling pathway and MAPK signaling pathway. Our works support that abnormal expression of nuclear Drosha was critical to tumor metastasis-related miRNA generation as previous findings in cervical SCC cells ${ }^{17}$ and Drosha cKO mice. ${ }^{18}$

miRNA-622 and miRNA-197 expressions were dysregulated and were closely related to tumor cell invasion in GCs. Using bioinformational and experimental evidence, we confirmed that $L A M C 2$ was a miR-622 target, and CD82, a miR-197 target. LAMC2 (laminin $\gamma 2$ ) is the only monomeric chain that was secreted from laminin 332 , consisting of the $a 3, \beta 3$ and $\gamma 2$ chains. Higher levels of LAMC2 are in most of the malignant tumors and correlate with poor overall survival and metastasis, as a therapeutic target for human cancers, ${ }^{19,20}$ whereas $C D 82$ (also called $K A /-1$ ), as a metastasis suppressor gene, is downregulated in cancers. ${ }^{21}$ These data support our findings that enhanced LAMC2 and decreased CD82 in GC cells are critical for gastric tumor invasion and metastasis.

Activation of EGFR-ERK1/2-MMP7 signaling axis regulated by nuclear Drosha may have a key role in GC cell invasion. EGFR and its downstream signaling have a critical function in gastric carcinoma development, and targeting EGFR may be a major strategy in the personalized treatment of gastrointestinal tumors. $^{22}$ The amplified EGFR is a worse factor in GC initiation, invasion and metastasis. ${ }^{23}$ LAMC2 colocalizes with EGFR to phosphorylate EGFR and activates its downstream signaling ERK1/2. ${ }^{12,24}$ CD82 suppresses EGFR expression and phosphorylation in EGF- and HGF-dependent manner in Hepa1-6 cells. ${ }^{15}$ Here we observed that the high level of nuclear Drosha decreased miR-622 or increased miR-197 to lead to an upregulated LAMC2 and downregulated CD82 in malignant GC cells. Knockdown of LAMC2 (or rescue of miR-622) expression or knockdown of miR-197 (or rescue of CD82) expression in MGC-803 cells inhibited tumor cell 
a

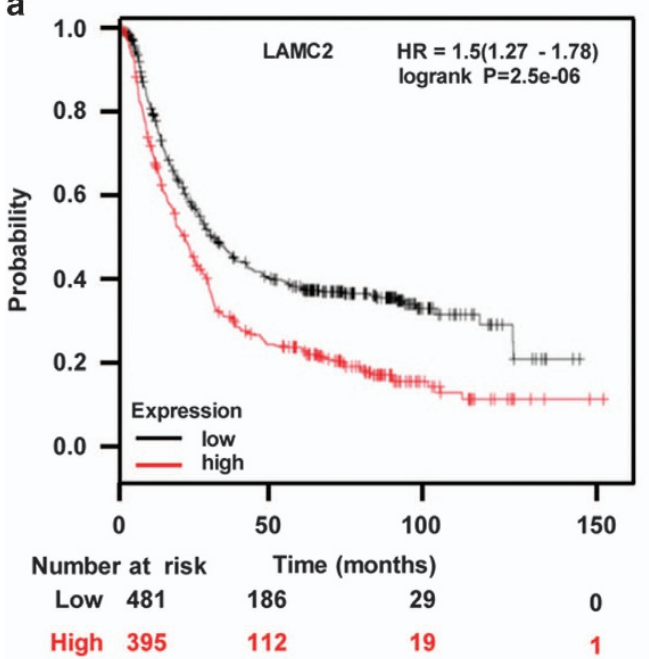

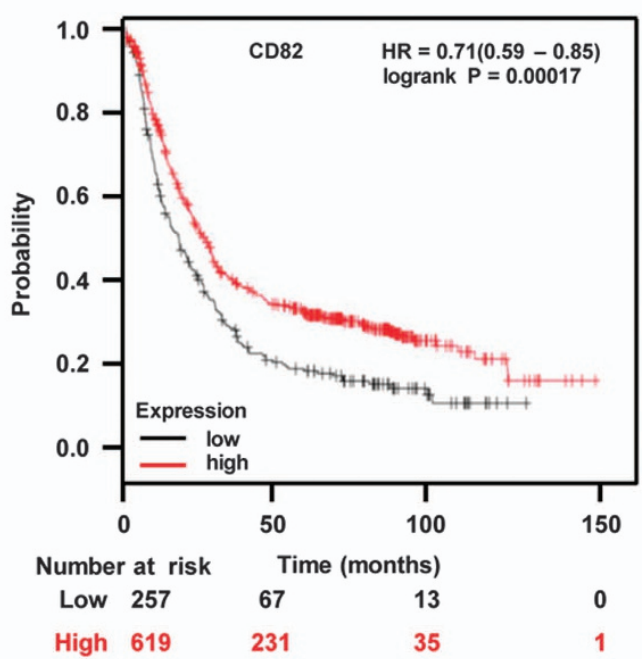

b
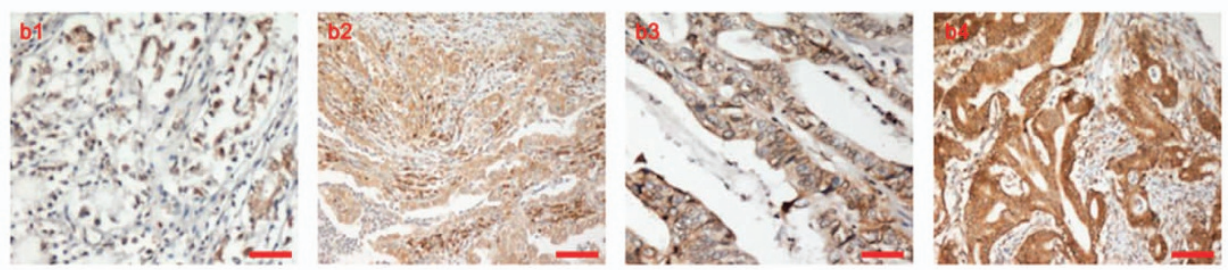

C
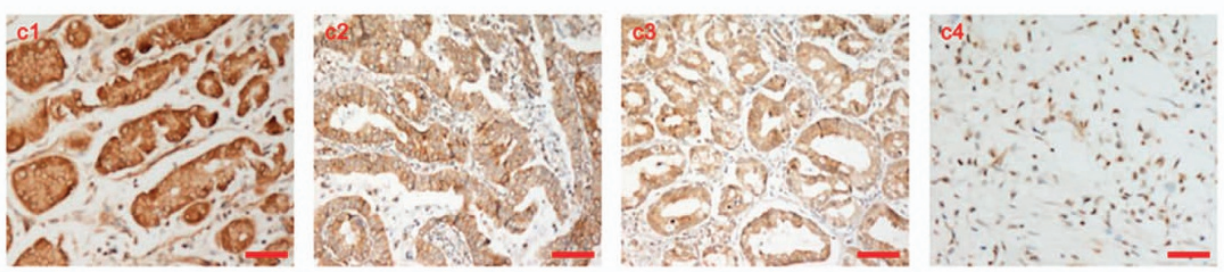

d
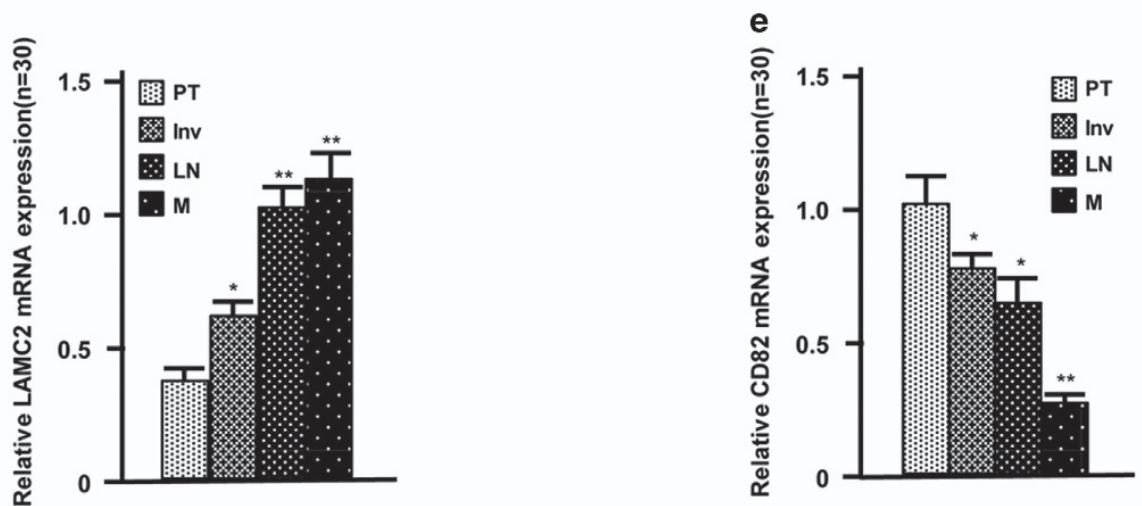

Figure 5 The enhanced LAMC2 and decreased CD82 indicate tumor metastasis and worse outcome of GC. (a) The overall survival of GC patients was evaluated by KaplanMeier plots. (b) The gradual increase of LAMC2 proteins were examined by IHC staining in GC specimens from the tumor in situ (b1), invasive tumor tissues (b2), tumors with lymph node metastasis (b3) and metastasis tissues (b4). (c) IHC staining to show the gradual decrease of CD82 proteins in GC tissues derived from the tumor in situ (c1), invasive tumors (c2), tumors with lymph node metastasis (c3) and tumors with distant metastasis locus (c4). (d and e) The expressions of LAMC2 (d) and CD82 (e) in various gastric carcinomas were quantitatively determined by qRT-PCR. PT: tumor in situ; Inv: invasive tumor; LN: tumor with lymph node metastasis; M: tumor with distant metastasis locus $\left({ }^{*} P<0.05,{ }^{*} P<0.01\right.$, versus PT). Scale bars, $100 \mu \mathrm{m}$

invasion. Inversely, the cell-invasive potential could be partially restored in Drosha-silenced MGC-830 cells. However, the reason why Drosha processes miR-622 and miR-197 differently is still unknown.
In summary, the current study reveals that nuclear Drosha may involve in the biogenesis of a set of metastasis-related miRNAs in GCs. Higher levels of miR-197 via downregulation of CD82 in coordination with lower levels of miR-622 via upregulation 

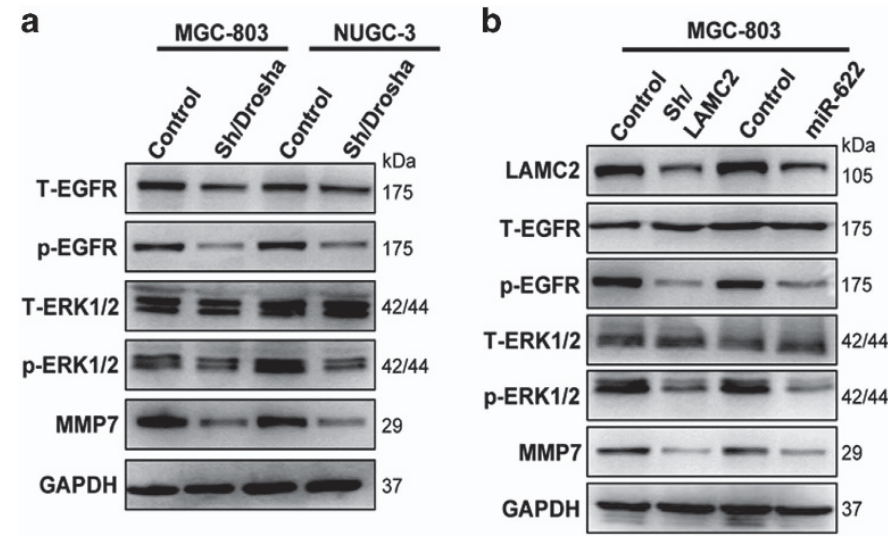

d

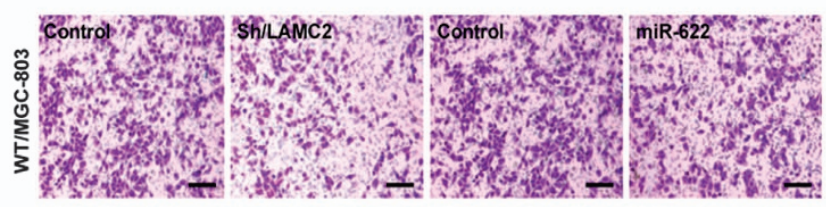

e

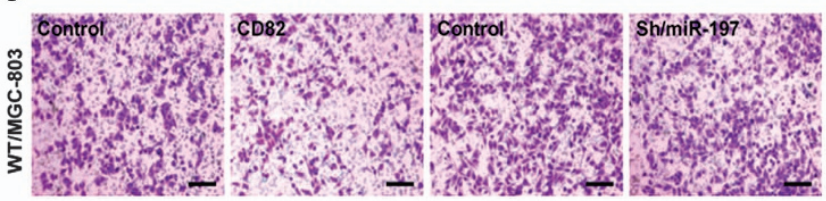

h

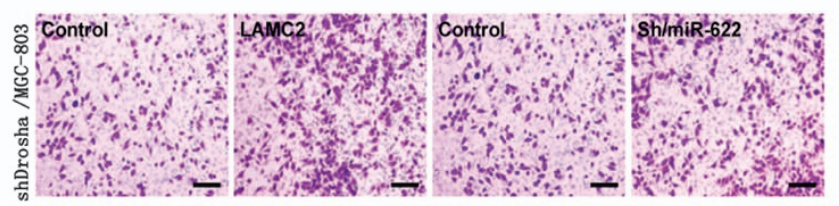

i

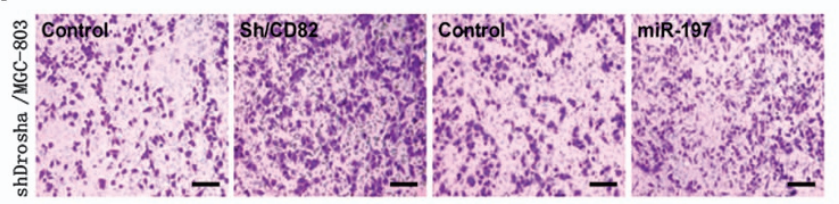

C

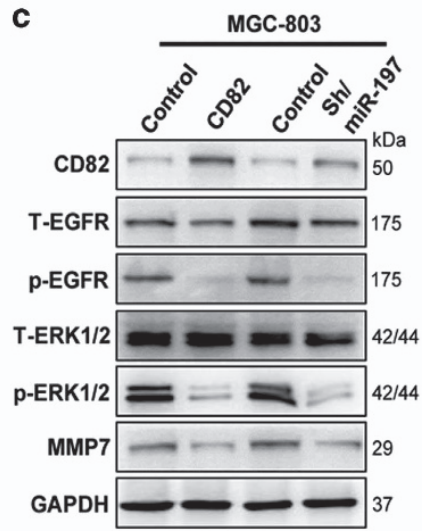

f

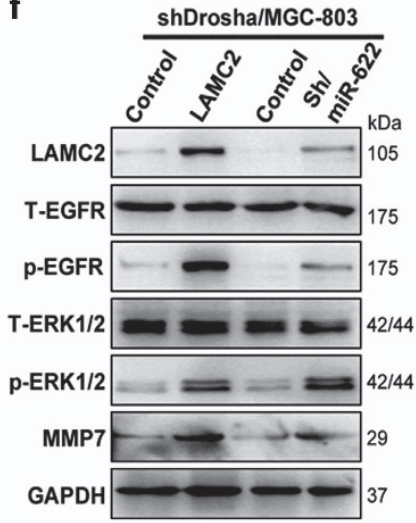

g

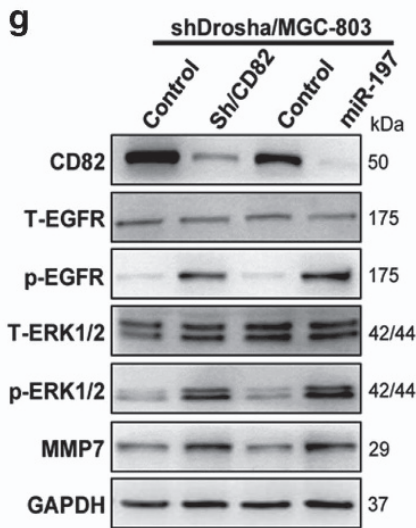

Figure 6 The enhanced LAMC2 orchestrates with decreased CD82 to promote tumor cell invasion via an EGFR-ERK1/2-MMP7 pathway. (a) The activated EGFR and ERK1/2 were detected in Drosha wild-type (WT) MGC-803 and NUGC-3 cells by western blotting. (b) The decreased p-EGFR and inactivation of ERK1/2 and lower levels of MMP7 were determined by western blotting in LAMC2-knockdown or miR-622-overexpressing MGC-803 cells. (c) Rescue of CD82 or knockdown of miR-197 in MGC-803 cells, and western blotting was used to determine the indicated protein expressions. (d and e) Cell invasion of MGC-803, as in (b) and (d), was tested using Transwell chambers. (f) Rescuing LAMC2 expression or stably knocking down miR-622 expression in Drosha-silenced MGC-803 cells, p-EGFR, p-ERK1/2 and MMP7 were determined by western blotting. (g) Western blotting to detect the indicated protein expressions in the MGC-803 cells with Drosha knockdown. ( $\mathrm{h}$ and $\mathrm{i}$ ) The cell-invasive potential was tested using the Transwell chamber for Drosha-silenced MGC-803 cells, as in (f) and (g). Glyceraldehyde 3-phosphate dehydrogenase (GAPDH) worked as a loading control in all western blotting analysis experiments

LAMC2 in GC activate EGFR-ERK1/2 signaling (Figure 7), thus having a role in promoting tumor cell invasion and metastasis.

\section{Materials and Methods}

Tissue specimens. Human gastric tumor tissues and their corresponding precancerous tissue were obtained from patients with gastric tumor undergoing surgery at the first affiliated hospital of Chongqing Medical University. None of the patients had previously undergone radiotherapy or chemotherapy treatment. All tissues included 20 normal adjacent tissue samples, 20 tissues derived from the tumor in situ, 65 lymph node metastasis tissue samples ( $N 0=20, N 1=15, N 2=15$ and $\mathrm{N} 3=15)$ and 20 distant metastasis tumor tissues $(\mathrm{M})$. The investigation was approved by the ethics committee of the Chongqing Medical University. 


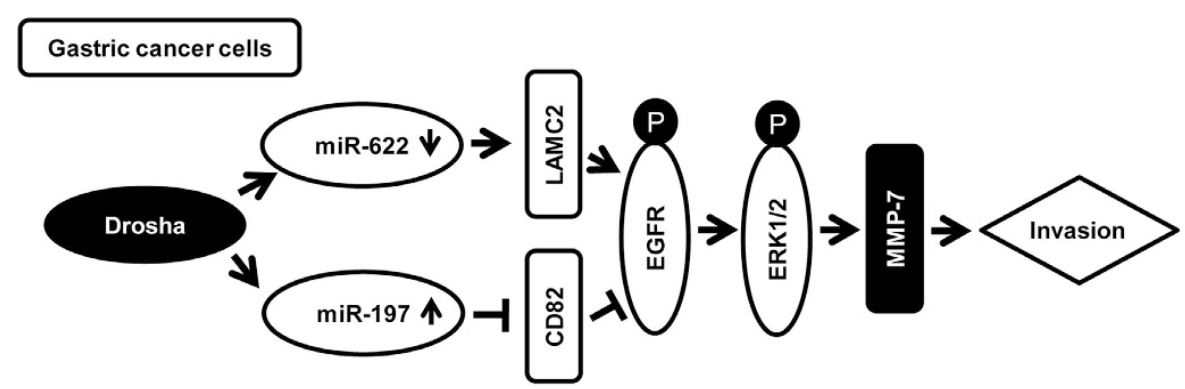

Figure 7 A model depicts the role of Drosha promoting GC cell invasion. Drosha activates EGFR-ERK1/2 signaling to promote GC cell invasion via regulation of miR-197, miR-622 and their targets LAMC2 and CD82

Cell culture. Human GC cell lines MKN-28 and HGC-27 and human normal gastric epithelial cell line GES-1 were obtained from the Shanghai Cell Bank of the Chinese Academy of Sciences (Shanghai, China). MGC-803, NUGC-3 and NCL-87 cell lines were kindly donated by Prof. Yang Ke of the Beijing Institute of Cancer Research (Beijing Shi, China). MGC-803 and GES-1 were maintained in DMEM containing 10\% FBS (Gibco-BRL, Australia); NUGC-3, HGC-27, MKN-28 and NCL-87 were routinely cultured in RPMI-1640 (Gibco) with 10\% FBS. All cells were incubated in a humidified atmosphere of $5 \% \mathrm{CO}_{2}$ at $37^{\circ} \mathrm{C}$.

Plasmid construction, inhibitors and mimics. The pHBLV-LAMC2 and CFP-CD82 plasmids were purchased from Hanbio Biotechnology Co. (Shanghai, China) or Addgene Inc. (http://www.addgene.org/). All the synthetic shRNA oligonucleotides used in this study were obtained from GenePharma (Shanghai, China). The transfection of shRNAs was performed using the Lipofectamine 2000 reagent (Invitrogen, Carlsbad, CA, USA). The pMIR-LAMC2- and pMIR-CD82Report vector were obtained by inserting wild-type and mutant binding sites of miR-622 or miR-197 in the 3'-UTR of LAMC2 or CD82 into the pMIR-Report vector (Ambion, Austin, TX, USA) at the Spel and Hindll sites. Mimics and inhibitors of miR-622, miR-197 and their controls were purchased from GenePharma (Shanghai, China). The sequences described above are provided in Supplementary Table 1.

Immunofluorescence. IF assay was performed as described previously. ${ }^{25}$ The primary antibody against Drosha (1:200; no. 12286; Abcam, Cambridge, MA, USA) or IgG control, and FITC-labeled goat anti-rabbit secondary antibody (1:200; Sigma, St. Louis, MO, USA) were used in IF. Cell nucleus was stained with DAPI. Immunofluorescent images were captured using a Nikon Eclipse 80i microscope (Eclipse 80i, Tokyo, Japan).

Immunohistochemistry. Immunohistochemical staining was performed as described previously. ${ }^{26}$ Briefly, the deparaffinized tissue sections $(4 \mu \mathrm{m})$ were heated for antigen retrieval, quenched for endogenous peroxidase activity and blocked with goat serum; the primary antibodies against Drosha (1:150; ab12286; Abcam), LAMC2 (1:150; Millipore, Darmstadt, Germany), CD82 (1:150; CST, Danvers, MA, USA), and secondary antibody (1:100; ZSBIO, Beijing, China) were used. After staining with diaminobenzidine and hematoxylin, the images were captured and assessed by the Image-Pro plus 6.0 software (Media Cybernetics, Rockville, MD, USA) to quantify the immunohistochemical staining. The mean optical density in 50 randomly selected areas (MOD $=1 O D /$ area) was used to evaluate the levels of protein expression.

The pathology scoring of the tissues was performed as described previously. ${ }^{26}$ Nuclear expression of Drosha was graded from $1+$ to $3+(1+, 1-25 \% ; 2+, 26-50 \%$; $3+$ and $>50 \%$ nuclear staining). The staining intensities of Drosha were assessed by examining $80 \%$ of the cell population. Images were captured using a Nikon Eclipse 80i microscope (Eclipse 80i).

RNA extraction and qRT-PCR. Total RNA was isolated using TRlzol (Invitrogen) routinely. The quantitative real-time PCR was performed in triplicate using SYBR Premix Ex Taq II (RR820A; TaKaRa, Dalian, China). Relative gene expression was measured as $2^{\mathrm{Ct}(\text { internal control) }-\mathrm{Ct}(\text { gene) }}$. The primers used are listed in Supplementary Table 2.

Western blot analysis. Nuclear and cytoplasmic extracts were prepared using a Nuclear and Cytoplasmic Protein Extraction Kit (Beyotime Institute of
Biotechnology, Jiangsu, China) as described previously. ${ }^{11}$ Total proteins were extracted using RIPA lysis buffer. Fifty micrograms of cell lysates were electrophoresed with $10 \%$ SDS-PAGE. The specific primary antibodies used against each protein in the immunoblotting were as follows: anti-Drosha (1:1000; Abcam), anti-PCNA (1:800; Abcam), anti-LAMC2 (1:1000; Millipore), anti-CD82 (1:1000; CST); the antibodies anti-EGFR, anti-p-EGFR, anti-ERK1/2, anti-p-ERK1/2, antiMMP7 and anti-GAPDH (1:1000; all from Beyotime); anti- $\beta$-tubulin (1:500; Santa Cruz Biotechnology, Santa Cruz, TX, USA) and anti- $\beta$-actin (1:1000; Boster, Wuhan, China). The appropriate horseradish peroxidase-conjugated secondary antibodies were subsequently applied. The proteins were visualized by the enhanced chemiluminescence system (Amersham, Pharmacia Biotech, Freiburg, Germany).

Wound healing and cell invasion assays. The experimental procedures of wound healing assays and cell invasion assay was described in detail previously. ${ }^{25}$ The NUGC-3 and MGC-803 cells were used in these assays. The width of scratches was recorded by phase contrast microscopy (Nikon TE2000-U; Nikon Corporation, Tokyo, Japan) and was measured using the Image $\mathrm{J}$ software (Rawak Software, Inc, Germany) at the designated time point. The percentage of the non-healed scratched area (S) for each replicate was calculated as follows: \% of the non-healed scratched area $=(\mathrm{S}$ (designed time) $/ \mathrm{S}$ (starting time $)) \times 100 \%$. The invaded cells for invasion assay were counted in five of the randomly selected fields. The data represent three experiments, each in triplicate (mean \pm S.E.).

miRNA array assay and bioinformatics analysis. The miRNA array assay was performed as described previously in detail. ${ }^{27}$ The MGC-803/DroshashRNA and MGC-803/NC-shRNA control cells were used in the Agilent miRNA array (Agilent, Santa Clara, CA, USA). The raw data were normalized by Quantile algorithm using the Gene Spring Software12.6 (Agilent, Santa Clara, CA, USA). These miRNAs were then grouped using hierarchical clustering with 'complete' using Heatmap.2 function (gplots package v.2.9.0).

The potential target genes for the dysregulation miRNAs were predicted using TargetScan v.6.2 algorithms, miRanda algorithms and DIANA-microT algorithms software (WWW.microrna.gr/WebServer) as described previously. ${ }^{27}$ All the copredicted target genes were used for subsequent functional analysis through DAVID Bioinformatics Resources 6.7. The $P$-value cutoff was set below 0.05 .

Luciferase reporter assay. Luciferase assays were performed as described previously. ${ }^{28}$ MGC- 803 cells were co-transfected with a total $800 \mathrm{ng}$ of miR-622, miR-197 expression plasmid or its control vector and the pMIR-LAMC2, pMIR-CD82 report vector (wild-type or mutant) or control vector using Lipofectamine 2000 (Invitrogen). pRL-TK worked as an internal control. The Renilla and firefly luciferase activities were measured by a Dual-Luciferase Reporter System (E1910; Promega, Madison, WI, USA) according to the manufacturer's instructions.

Statistical analysis. Statistical analysis was performed using the SPSS standard version 13.0 software (Chicago, IL, USA). The data of three independent experiments were presented as the mean \pm S.D. The independent Student's $t$-test was used to compare the continuous variables between two groups. A $P$-value $<0.05$ was considered to be statistically significant.

\section{Conflict of Interest}

The authors declare no conflict of interest. 
Acknowledgements. This work was supported, in part, by National Natural Science Foundation of China (NSFC 81172296, NSFC 81472476 and NSFC 81402180).

1. Jemal A, Bray F, Center MM, Ferlay J, Ward E, Forman D. Global cancer statistics. $C A$ Cancer J Clin 2011; 61: 69-90.

2. Lam EK, Wang $X$, Shin VY, Zhang S, Morrison $\mathrm{H}$, Sun $\mathrm{J}$ et al. A microRNA contribution to aberrant Ras activation in gastric cancer. Am J Transl Res 2011; 3: 209-218.

3. Chen K, Yang D, Li X, Sun B, Song F, Cao W et al. Mutational landscape of gastric adenocarcinoma in Chinese: implications for prognosis and therapy. Proc Natl Acad Sci USA 2015; 112: 1107-1112.

4. Wan $X$, Ding $X$, Chen $S$, Song $H$, Jiang $H$, Fang $Y$ et al. The functional sites of miRNAs and IncRNAs in gastric carcinogenesis. Tumour Biol 2015; 36: 521-532.

5. Shu GS, Yang ZL, Liu DC. Immunohistochemical study of Dicer and Drosha expression in the benign and malignant lesions of gallbladder and their clinicopathological significances. Pathol Res Pract 2012; 208: 392-397.

6. Díaz-García CV, Agudo-López A, Pérez C, López-Martín JA, Rodríguez-Peralto JL, de Castro $\mathrm{J}$ et al. DICER1, DROSHA and miRNAs in patients with non-small cell lung cancer: implications for outcomes and histologic classification. Carcinogenesis 2013; 34: 1031-1038.

7. Farazi TA, Hoell Jl, Morozov P, Tuschl T. MicroRNAs in human cancer. Adv Exp Med Biol 2013; 774: 1-20

8. Chi Y, Zhou D. MicroRNAs in colorectal carcinoma - from pathogenesis to therapy. J Exp Clin Cancer Res 2016; 35: 43.

9. Bertoli G, Cava C, Castiglioni I. MicroRNAs: new biomarkers for diagnosis, prognosis therapy prediction and therapeutic tools for breast cancer. Theranostics 2015; 5: 1122-1143.

10. Xu LF, Wu ZP, Chen Y, Zhu QS, Hamidi S, Navab R. MicroRNA-21 (miR-21) regulates cellular proliferation, invasion, migration, and apoptosis by targeting PTEN, RECK and Bcl-2 in lung squamous carcinoma, Gejiu City, China. PLoS One 2014; 9: e103698.

11. Zhang H, Hou Y, Xu L, Zeng Z, Wen S, Du YE et al. Cytoplasmic drosha is aberrant in precancerous lesions of gastric carcinoma and its loss predicts worse outcome for gastric cancer patients. Dig Dis Sci 2016; 61: 1080-1090.

12. Garg M, Kanojia D, Okamoto R, Jain S, Madan V, Chien W et al. Laminin-5 $\gamma$-2 (LAMC2) is highly expressed in anaplastic thyroid carcinoma and is associated with tumor progression, migration, and invasion by modulating signaling of EGFR. J Clin Endocrinol Metab 2014; 99: E62-E72.

13. Li Y, Huang X, Zhang J, Li Y, Ma K. Synergistic inhibition of cell migration by tetraspanin CD82 and gangliosides occurs via the EGFR or cMet-activated PI3K/Akt signalling pathway. Int J Biochem Cell Biol 2013; 45: 2349-2358.

14. Ha M, Kim VN. Regulation of microRNA biogenesis. Nat Rev Mol Cell Biol 2014; 15: 509-524.

15. Khoshnaw SM, Rakha EA, Abdel-Fatah T, Nolan CC, Hodi Z, Macmillan RD et al. The microRNA maturation regulator Drosha is an independent predictor of outcome in breast cancer patients. Breast Cancer Res Treat 2013; 137: 139-153.

16. Jafarnejad SM, Sjoestroem C, Martinka M, Li G. Expression of the RNase III enzyme DROSHA is reduced during progression of human cutaneous melanoma. Mod Pathol 2013; 26: $902-910$.

17. Muralidhar B, Winder D, Murray M, Palmer R, Barbosa-Morais N, Saini H et al. Functiona evidence that Drosha overexpression in cervical squamous cell carcinoma affects cell phenotype and microRNA profiles. J Pathol 2011; 224: 496-507.
18. Fan $\mathrm{P}$, Chen $\mathrm{Z}$, Tian $\mathrm{P}$, Liu $\mathrm{W}$, Jiao $\mathrm{Y}, \mathrm{Xue} Y$ et al. miRNA biogenesis enzyme Drosha is required for vascular smooth muscle cell survival. PLOS One 2013; 8: e60888.

19. Moon YW, Rao G, Kim JJ, Shim HS, Park KS, An SS et al. LAMC2 enhances the metastatic potential of lung adenocarcinoma. Cell Death Differ 2015; 22: 1341-1352.

20. Liu W, Tian F, Jiang P, Zhao X, Guo F, Li X et al. Aberrant expression of laminin $\gamma 2$ correlates with poor prognosis and promotes invasion in extrahepatic cholangiocarcinoma. J Surg Res 2014; 186: 150-156.

21. Feng J, Huang C, Wren JD, Wang DW, Yan J, Zhang J et al. Tetraspanin CD82: a suppressor of solid tumors and a modulator of membrane heterogeneity. Cancer Metast Rev 2015; 34: 619-633.

22. Rolfo C, Bronte G, Sortino G, Papadimitriou K, Passiglia F, Fiorentino E et al. The role of targeted therapy for gastrointestinal tumors. Expert Rev Gastroenterol Hepatol 2014; 8: 875-885.

23. Higaki E, Kuwata T, Nagatsuma AK, Nishida Y, Kinoshita T, Aizawa M et al. Gene copy number gain of EGFR is a poor prognostic biomarker in gastric cancer: evaluation of 855 patients with bright-field dual in situ hybridization (DISH) method. Gastric Cancer 2016; 19 : 63-73.

24. Guo B, Zhang T, Su J, Wang K, Li X. Oxymatrine targets EGFR (p-Tyr845) and inhibits EGFR-related signaling pathways to suppress the proliferation and invasion of gastric cancer cells. Cancer Chemother Pharmacol 2015; 75: 353-363.

25. Wang L, Hou Y, Sun Y, Zhao L, Tang X, Hu P et al. c-Ski activates cancer-associated fibroblasts to regulate breast cancer cell invasion. Mol Oncol 2013; 7: 1116-1128.

26. Yuan J, Liu M, Yang L, Tu G, Zhu Q, Chen M et al. Acquisition of epithelial-mesenchymal transition phenotype in the tamoxifen-resistant breast cancer cell: a new role for $G$ proteincoupled estrogen receptor in mediating tamoxifen resistance through cancer-associated fibroblast-derived fibronectin and $\beta 1$-integrin signaling pathway in tumor cells. Breast Cancer Res 2015; 17: 69 .

27. Zhao L, Sun Y, Hou Y, Peng Q, Wang L, Luo $\mathrm{H}$ et al. MiRNA expression analysis of cancerassociated fibroblasts and normal fibroblasts in breast cancer. Int J Biochem Cell Biol 2012; 44: 2051-2059.

28. Tang X, Hou Y, Yang G, Wang X, Tang S, Du YE et al. Stromal miR-200s contribute to breast cancer cell invasion through CAF activation and ECM remodeling. Cell Death Differ 2016; 23: 132-145.

(1) Cell Death and Disease is an open-access journal published by Nature Publishing Group. This work is licensed under a Creative Commons Attribution 4.0 International License. The images or other third party material in this article are included in the article's Creative Commons license, unless indicated otherwise in the credit line; if the material is not included under the Creative Commons license, users will need to obtain permission from the license holder to reproduce the material. To view a copy of this license, visit http://creativecommons.org/licenses/by/4.0/

(C) The Author(s) 2017

Supplementary Information accompanies this paper on Cell Death and Disease website (http://www.nature.com/cddis) 